\title{
EL PAPEL GEOESTRATÉGICO DE ASIA CENTRAL
}

\author{
Mauricio Lascurain Fernández
}

\section{INTRODUCCIÓN}

La década de 1990 fue una época de cambio para Asia Central. Por el inevitable agotamiento del modelo soviético, que llegaba a su fin en 1991, surgieron cinco nuevos estados independientes en la región: Uzbekistán, Turkmenistán, Kazajstán, Tayikistán y Kirguizistán. El resurgimiento del heartland del que hablaba Halford Mackinder ${ }^{1}$ forzaba a una nueva recomposición geopolítica y geoeconómica de Asia Central, de la cual participarían, inicialmente, las principales potencias regionales. Sin embargo, después de los acontecimientos del 11 de septiembre de 2001 (11/S), en Washington y Nueva York, la situación cambiaría radicalmente para la geopolítica de la región.

Como consecuencia de los ataques del 11/S, los Estados Unidos de América (EE.uU.) emprendieron la llamada "guerra contra el terrorismo" e intervinieron militarmente en Afganistán y, más tarde, en Irak, lo que les permitió, por primera vez en su historia, instalar bases militares en territorio exsoviético y posicionarse en una región estratégica por su ubicación (al encontrarse cerca de China, Rusia, el Golfo Pérsico, Pakistán e India) y por su abundancia de hidrocarburos. La participación de los EE.uU. ha complicado la dinámica geoestratégica de la región, al enfrentar a tres grandes potencias (EE.UU., Rusia y China) y diferentes actores regionales en una intensa rivalidad por influir en las repúblicas de Asia Central.

1 "The Geographical Pivot of History", The Geographical Journal, vol. 23, núm. 4, 1904, pp. 421-437. 
Por tanto, la desaparición de un mando central y la aparición de cinco nuevos estados con intereses particulares han dado como resultado una diversidad de conflictos que amenazan la estabilidad presente y futura de Asia Central, con implicaciones políticas, económicas y de seguridad, tanto para Europa y Asia, como también para los EE.UU.

Este artículo pretende identificar los conflictos más importantes en Asia Central y, con base en ello, determinar los posibles escenarios para la región. Para lograr lo anterior, el trabajo se ha dividido en cinco secciones. La primera consiste en analizar la transición política y geoestratégica que envuelve a Asia Central desde la década de 1990. La segunda trata el problema del Mar Caspio y sus consecuencias geopolíticas y geoeconómicas para la región y el resto del mundo. En la tercera se analizará el complicado sistema que encierra el Valle de Fergana y sus diferentes efectos en Asia Central. En la cuarta se hará un análisis de los diferentes actores regionales e internacionales que tienen interés por la región. En la quinta se ofrecerán algunas conclusiones generales.

\section{La transformación de Asia Central}

Entre los cambios más relevantes que se han vivido en Asia Central, el que descuella sobre los demás es, sin duda, la independencia y creación de cinco nuevos estados en la región después de la caída del sistema soviético. ${ }^{2}$ Sin embargo, el estatuto de estados independientes que adquirieron estos países, a principios de la década de 1990, se realizó por una situación muy diferente a la que se ha experimentado en otras partes del mundo. N. Sainz afirma que "la principal característica de esta nueva identidad es que dentro de los mismos países no existían movimientos políticos tan fuertes que exigiesen la independencia de la ex URss". ${ }^{3}$ Por tanto,

${ }^{2}$ Uzbekistán, Turkmenistán, Kazajstán, Tayikistán y Kirguistán.

3 "Asia Central en un mundo en cambio: de región periférica a área generadora de aplicaciones de políticas”, Revista CIDOB d'Afers Internacionals, núms. 7071, 2005, p. 118. 
el cambio a la independencia fue el resultado, más bien, del hundimiento político, ideológico y económico del centro (URSs), antes que la consecuencia de una voluntad expresada por la población de la periferia.

En este sentido, la transición de Asia Central se puede analizar por lo menos desde dos perspectivas. La primera se puede interpretar como la salida del modelo político soviético, con el objetivo de edificar una nueva formación nacional. La segunda es la transición geopolítica, toda vez que la fundación de nuevos estados independientes en una región estratégica propicia que ésta cobre importancia vital en el ámbito internacional por el potencial de sus recursos.

En lo que atañe al modelo político, se debe señalar que las repúblicas de Asia Central accedieron a su independencia, sin haberlo solicitado, lo cual influyó considerablemente en su evolución (a diferencia de las repúblicas europeas de la unss, la región Centroasiática estaba a favor de la unión con el fin de mantener su desarrollo), ya que los dirigentes del partido comunista local se convertirían en los nuevos líderes políticos de los Estados independientes. Djalili y Keller coinciden en que "en la ausencia de élites nacionalistas formadas en la lucha por la descolonización fueron los mismos miembros de la administración soviética quienes acapararon el poder". ${ }^{4}$ Aunque estas élites han demostrado ser capaces de adaptarse sorprendentemente a las nuevas circunstancias políticas y al nuevo aparato ideológico, continúan mostrando el mismo comportamiento represivo, que heredaron de su pasado soviético. ${ }^{5}$

Los gobiernos centroasiáticos han evolucionado desde un sistema totalitarista hasta sistemas políticos autoritarios con el predominio de un partido político, en los cuales el resto de los partidos prácticamente no tienen ningún poder opositor, por lo que la

4 "Regímenes políticos de Asia Central: crisis de legitimidad, violencia política y perspectivas inciertas”, Revista CIDOB d'Afers Internacionals, núms. 70-71, 2005, p. 39.

${ }^{5}$ M. L. Alonso, "Fundamentalismo Islámico en Asia Central: causas, actores y perspectivas de futuro", Grupo de Estudios Internacionales Contemporáneos, AI 014/ 2011, Asia-Pacífico, 14 de julio de 2011, pp. 3-14. 
expectativa de una auténtica alternancia política por medio de las urnas se encuentra muy lejos de la realidad de Asia Central. ${ }^{6}$ A más de dos décadas de la desintegración de la uRss, esta realidad impera todavía en la región centroasiática. Lo anterior revela una concentración de poder vuelta en represión por parte de los gobiernos en contra de su población, provocando, entre otros fenómenos, el surgimiento de movimientos fundamentalistas islámicos, en especial en la región del Valle de Fergana.

Autores como Djalili y Keller, Petrov y Kovalskii han supuesto que con el tiempo y el cambio generacional se observará una nueva élite formada después de la independencia de las repúblicas, la cual romperá por completo con el pasado soviético. ${ }^{7}$ Sin embargo, este cambio generacional podría resultar un fracaso político más para los países de la zona, ya que la ausencia de un sucesor adecuado, la falta de experiencia democrática o la carencia de coordinación entre los dirigentes locales, podrían retrasar el proceso democrático y agravar la precaria condición de los derechos humanos en la región, lo que llevaría a perder una oportunidad para que la región se integre al mundo.

Con respecto del cambio en la geopolítica, esto ha implicado la reaparición de una región en el tablero internacional del que había estado ausente desde su integración a la Unión Soviética. Los nuevos países centroasiáticos forman parte de lo que Halford Mackinder llamaba el "pivote geográfico de la historia" o heartland, cuya dominación garantizaría, a la larga, el control del mundo. ${ }^{8}$ La entrada en la escena internacional una vez más de esta

${ }^{6}$ B. R. Rubin, "Central Asia Wars and Ethnic Conflicts, Rebuilding Failed States”, Human Development Report Office, United Nations Development Programme, 2004.

${ }^{7}$ Cf. Djalili y Keller, art. cit.; N. I. Petrov, "Political Stability in the Conditions of the Command-Administrative Regime", y V. F. Kovalskii, "Democratic Declarations and Political Realities", ambos en A. Vassiliev (ed.), Central Asia, Political and Economic Challenges in the Post-Soviet Era, Londres, Saqi Books, 2001. A pesar de que estas predicciones datan desde principios de 2000, en 2015 siguen vigentes, ya que no ha tenido lugar la transición política en los países de Asia Central, con la excepción de Kirguistán, pero sin evidenciar una mayor trascendencia para la región.

${ }^{8}$ Art. cit. 
zona tan influyente, constituye un fenómeno de alcance excepcional, por su ubicación e interés geoestratégico para las potencias regionales como China, Irán o la misma federación rusa, que por medio de la denominada Unión Económica Euroasiática, que entró en vigor el 1 de enero de 2015, pretende retomar el control de la región.

Esta transformación geopolítica ha traído consigo viejos problemas, como el del estatuto jurídico del mar Caspio, así como una serie de nuevas dificultades políticas, sociales y económicas para los países de la región, al igual que para aquellos países ajenos a la zona (EE.UU., Unión Europea, Turquía y Japón), que intentan modificar el mapa geopolítico a su favor.

\section{El problema EN EL CASpio}

Con la desintegración de la uRss, el interés por el mar Caspio aumentó, ya que a lo largo de sus costas se habían constituido nuevos estados independientes, los cuales reclamaban su derecho a utilizar los recursos de éste, según conviniera a cada cual. La explotación y exploración internacionales de las reservas de hidrocarburos, así como su salida hacia los mercados internacionales, obligaban a la necesidad de un nuevo estatus jurídico del mar; sin embargo, la falta de éste ha suscitado diferentes disputas entre los países ribereños.

La independencia de los países del Cáucaso y de Asia Central, en la década de 1990, llamó la atención de los países occidentales, así como de las principales empresas privadas y estatales productoras de energía del mundo: Royal Dutch Shell, Arawak Energy Ltd., BP, ExxonMobiel, Chevron, Lukoil, Surgutneftegaz, Gazprom, ConocoPhillips, Dragon Oil, Chinese National Petroleum Company, Petronas, entre otras, por tener algunas de las mayores reservas de gas en el mundo, así como importantes reservas de petróleo. ${ }^{9}$

${ }^{9}$ Cf. EIA, Overview of oil and natural gas in the Caspian Sea region, Caspian Sea series, 26 de agosto, 2013a, pp. 6-7, y Beta: International, Reserves and Capacity, Petroleum and Natural Gas, 2016, en http:/ / www.eia.gov/beta/international/, consulta- 
Geográficamente, el mar Caspio se conforma de varios estados ribereños: Azerbaiyán, Irán, Rusia, Kazajstán y Turkmenistán, además de Georgia, que está involucrada en la salida de los hidrocarburos hacia los mercados internacionales. El mar Caspio, en cuanto a su geopolítica, representa un escenario de conflicto entre las diferentes potencias, porque en él se libra una batalla sin igual por el control de los oleoductos y por la explotación de los hidrocarburos. ${ }^{10}$ Por ejemplo, Moscú ve con recelo los planes de Turkmenistán de construir nuevos oleoductos para transportar el energético principalmente a Europa sin tener que usar las tuberías rusas. Esta situación se agravó aún más, cuando, en 2009, tras la ruptura de un extremo de la tubería que transporta el gas turkmeno, se obstaculizó la exportación de éste a Rusia (por entonces su principal cliente), lo que llevó al agravamiento de las relaciones con la federación rusa. En estas circunstancias, Moscú ha tratado de evitar resolver el estatus jurídico del mar Caspio, lo que se ha reflejado en una disminución en el flujo de energéticos de los estados ribereños por medio de esta fuente de recurso. ${ }^{11}$

Durante el periodo soviético, el Caspio había sido considerado por la URss e Irán como un lago regido por tratados bilaterales y por la legislación interna de cada uno de los países, por lo que los recursos eran de propiedad conjunta y exclusiva para ambos países; asimismo se regulaba la explotación de los recursos pesqueros, sin delimitar fronteras marítimas de una manera clara. ${ }^{12}$

$\mathrm{Al}$ desaparecer la Unión Soviética, la situación cambió drásticamente. El número de países ribereños pasó de dos a cinco y las intenciones sobre el uso del mar variaban de un país a otro. Hasta

do en julio de 2016. Según esta última fuente, las principales reservas en 2015 de petróleo crudo de los países de Asia Central ribereños del Mar Caspio se encuentran Kazajstán (30 mil millones de barriles), mientras que las reservas de gas natural se concentran en Turkmenistán (265 trillones de pies cúbicos).

${ }^{10}$ Cf. EIA, Caspian countries are developing new oil and natural gas export capacity, 2013b, en http://www.eia.gov/todayinenergy/detail.cfm?id=12931, consultado en noviembre de 2015 .

${ }^{11}$ MAEc, "Turkmenistán, ficha país", Gobierno de España, Oficina de Información Diplomática, 2013.

12 Sainz, art. cit., pp. 119-120. 
2015, los países ribereños no han podido llegar a un acuerdo sobre el estatuto jurídico ni sobre la delimitación de fronteras marítimas, principalmente porque hay dos versiones diferentes de cómo explotar los recursos del Caspio: la primera se refiere a que éste es un lago y la segunda, a que es un mar.

Según la posición que se asuma respecto al Caspio, las consecuencias jurídicas son diferentes. En el caso de definirse como mar -y si los estados concuerdan en trasladar a mares cerrados, como es el caso del Caspio, el derecho internacional aplicable a mares abiertos- los estados ribereños dispondrán, además de sus aguas territoriales de una anchura máxima de doce millas marinas, de una zona económica exclusiva en la que podrán ejercer sus derechos soberanos de exploración y explotación de las aguas, los fondos marinos y el subsuelo. ${ }^{13} \mathrm{El}$ principio adoptado sería el de reparto de recursos.

En el caso de considerar el Caspio como un lago, los estados ribereños dispondrán de una zona de diez millas marinas. Más allá de esa zona, "deberán explotar los recursos sobre la base de un acuerdo común”. La explotación de los recursos estará bajo el control de una autoridad internacional que reúna a los países ribereños y cuyas decisiones deberán tomarse unánimemente. ${ }^{14}$

Ante estas dos posiciones sobre el uso del Caspio, los estados ribereños han cambiado su punto de vista según el estatuto jurídico. Rusia e Irán han utilizado la definición de lago, ya que implica una explotación concertada de recursos, sin que ninguna de las partes tenga exclusivo derecho de propiedad más allá de su zona. En ese sentido, M. A. Pérez afirma que Rusia e Irán prefieren la definición de lago o régimen de condominio, ya que "los canales del Volga y el Don, que conectan con otros mares (tal y como lo plantean Kazajistán y Azerbaiyán), no están sometidos a las leyes internacionales de navegación aprobadas en 1982, ya que son propiedad rusa y por lo tanto no cumplen la función de salida natural

13 onu, Convención de las Naciones Unidas sobre el Derecho del Mar, 2006, en http.//www.un.org/depts/los/convention_agreements/texts/unclos/convemar_es.pdf

14 Sainz, art. cit., p. 120. 
a otros mares". ${ }^{15}$ Por un lado, Rusia e Irán manifiestan que la legislación internacional no habla específicamente sobre las limitaciones que debe tener un lago, a condición de poder ser explotado. Por otro, Kazajstán, Azerbaiyán y, en menor medida Turkmenistán, ven el Caspio como mar interno. Estos países consideran que "la definición de mar se ajusta a las características físicas del Caspio, ya que los canales Volga y Don conectan al Caspio con los mares Negro y Báltico" 16 y, por tanto, estiman que se debe observar la legislación internacional para la administración del Caspio como mar.

Es evidente que la postura de cada uno de los países varía según sus intereses. Sin embrago, se ha tratado de realizar varios esfuerzos para definir el estatuto legal del Caspio, sin que hasta el momento haya un veredicto final. Por ejemplo, en el año 2003 los gobiernos ruso, azerí y kazaja hicieron una propuesta para dividir el fondo del Caspio en sectores nacionales (siguiendo el trazado de la línea mediana, mientras las aguas superficiales siguen siendo propiedad común de los estados ribereños), la cual permite la extracción en la actualidad. Con todo, el gobierno iraní ha rechazado tal propuesta, ya que sería el gran perdedor en términos de porcentaje del reparto de las aguas. Según esa propuesta, a Irán correspondería aproximadamente 13\%, en compensación con el casi $20 \%$ que tocaría a los otros estados. ${ }^{17}$

Además de éstos, hay otros motivos por los que no hay un acuerdo entre los países. Uno de ellos se debe a las diferentes acciones unilaterales de los países ribereños. A propósito, Pérez llega a la conclusión de que

la existencia de grandes reservas de petróleo y gas en el subsuelo marino del Mar Caspio ha sido considerada por Azerbaiyán, Turkmenistán y Kazajstán como la única base de su desarrollo económico a corto plazo. Al ser una prioridad absoluta para estos gobiernos se

15 "El status jurídico del Mar Caspio", Geoeconomics and Geopolitics Journal, 1994, p. 7.

${ }^{16}$ Loc. cit.

17 Sainz, art. cit., p. 120, y R. M. Cutler, "Developments in the evolving Caspian legal regime: part 2-2”, FSU Oil Ẽ Gas Monitor, núm. 97, 2000, pp. 4-6, en http://www.gasandoil.com/goc/news/ntc03767.htm 
han lanzado a la carrera por explotar, perforar los fondos marinos y construir plataformas petrolíferas dentro de aquellas zonas marítimas que consideran bajo su jurisdicción. ${ }^{18}$

Asimismo, Pérez afirma que Rusia e Irán pretenden continuar con la hegemonía que han venido ejerciendo desde antes de la independencia de las nuevas repúblicas exsoviéticas, por lo que un acuerdo de manera multilateral es poco probable, por lo menos en el corto plazo. ${ }^{19}$

De lo hasta aquí analizado, se deduce que la falta de un acuerdo multilateral, para el manejo de los recursos en el Caspio, es un foco de inestabilidad en Asia Central. Esa incertidumbre llamaría la atención de las grandes potencias mundiales, para imponer un nuevo orden, que no convendría a las nuevas repúblicas, ni a Rusia ni a Irán.

En constantes reuniones, como la tenida en Rusia en 2014, los países ribereños del Caspio han tratado de implementar acuerdos que favorezcan sus intereses en la región, a más de llevarse a cabo reuniones multilaterales y de formalizarse una cumbre encaminada a tratar el tema sobre el mar Caspio. Por ejemplo, se ha tratado en especial el estatuto jurídico del Caspio, así como temas de seguridad. Para ello, los jefes de Estado firmaron en 2014 un Acuerdo de Cooperación en el campo de la hidrometeorología, así como el Convenio sobre la Cooperación en el campo de la prevención y eliminación de las situaciones de emergencia en el mar Caspio, y celebraron la Convención sobre la conservación y el uso racional de los recursos biológicos marinos del Caspio. ${ }^{20} \mathrm{Sin}$ embargo, el estatuto jurídico del mar Caspio en los últimos años (2014-2015) no dista del establecido en los años noventa y principios del siglo xxi por la constante lucha de intereses entre los países de la región. Además, un elemento que se añade a este conflicto es el de la explotación de los hidrocarburos.

18 Art. cit., p. 7.

${ }^{19}$ Loc. cit.

20 "Cuarta Cumbre del Caspio", Kremlin, Astrakhan, 2014, http://kremlin. $\mathrm{ru} / \mathrm{news} / 46686$, consultado en septiembre de 2014. 
En este sentido, desde principios de 1990, toda la cuenca del Caspio ha sido tema de discusión de las grandes compañías petroleras y de sus gobiernos, a causa de la supuesta importancia de sus reservas de hidrocarburos. Varias fuentes han estimado que las reservas de Asia Central podrían ser tan grandes que en un futuro serían el perfecto sustituto del Golfo Pérsico. Sin embargo, es preciso señalar que las publicaciones referentes a las reservas petrolíferas y a la importancia de la zona también podrían ser parte de una estrategia política, de la que participan los principales países consumidores de hidrocarburos para presionar a los países productores. ${ }^{21}$

Las reservas agregadas de petróleo en estos cuatro países de Asia Central y el Cáucaso Sur se han multiplicado por 5 en los últimos 15 años, pasando de 7,7 miles de millones de barriles, en 1998, a 42 miles de millones, en 2015. En relación con el gas natural, encontramos que las reservas en la zona han pasado de 236 trillones de pies cúbicos en 1998 a 419 trillones en 2015, pasando de $4 \%$ hasta $11,4 \%$ sobre el total de reservas mundiales en este periodo. ${ }^{22}$

A pesar de las diferentes estimaciones sobre las reservas probadas de la región, las compañías occidentales (Chevron, Exxon/Mobile, Shell, BP, etc.) poseen hoy en día aproximadamente el $60 \%$ de las reservas de la zona, mientras que el resto permanece en manos de las compañías paraestatales de los estados ribereños, lo que demuestra que, desde un punto de vista geopolítico, los occidentales ya han conseguido una importante penetración en la región, con la finalidad de asegurar el suministro futuro de hidrocarburos. ${ }^{23}$

El National Energy Policy Development Group ${ }^{24}$ considera que en el año 2020 la región del Golfo representará por sí sola entre 54

${ }^{21} \mathrm{~J}$. Laherrere, "Forecasting future production from past discovery", OPEC Semminar, septiembre 28-29, Vienna, 2001.

22 Cf. BP, "Statistical Review of World Energy", June 2014, en http://www.bp. $\mathrm{com} /$ content/dam/bp/pdf/Energy-economics/statistical-review-2014/BP-statistical-review-of-world-energy-2014-full-report.pdf, consultado en octubre de 2014, y EIA, Beta: International Reserves and Capacity, Petroleum and Natural Gas, 2016, en http:/ / www.eia.gov/beta/international/, consultado en julio de 2016.

${ }^{23}$ Cf. O. Roy, La nueva Asia Central o la fabricación de naciones, Madrid, Sequitur, 1997, y J. Laherrere, art. cit.

24 Reliable, Affordable, and Environmentally Sound Energy for America's Future, Washington, DC, United States Government Printing, 2001. 
y $67 \%$ de las exportaciones petrolíferas mundiales y garantizará el $100 \%$ de las exportaciones mundiales de petróleo hacia el año 2050. Por tanto, el litoral del Caspio podría convertirse en una fuente de aprovisionamiento suplementaria que permita diversificar temporalmente las importaciones de hidrocarburos de Europa y Sureste asiático, por lo que será necesario ser prudentes respecto a las capacidades suministradoras del mar Caspio. En estas circunstancias, los estados de Asia Central, como Turkmenistán, han expresado su intención de sumarse a las iniciativas principalmente europeas con su inclusión en el proyecto del gasoducto Transcaspio para abastecer a Europa. ${ }^{25}$

Por lo que toca al transporte de los hidrocarburos, existen varios oleoductos y gasoductos que bordean y cruzan Asia Central. Sin embargo, algunos países centroasiáticos se encuentran en la disyuntiva de cómo encontrar una salida para los hidrocarburos hacia el mercado internacional. En la vasta gama de oleoductos que actualmente están funcionando se encuentran: el Uzen-Atyrau-Samara, el Bakú-Novorossiysk (Northen Route Export Pipeline), el BakúSupsa (Western Route Export Pipeline) y el Caspian Pipeline Consortium (CPC), el cac Pipeline System, el Kazakhstan-China Oil Pipeline. Sin embrago, hay otros ductos para sacar al mercado internacional los hidrocarburos del Caspio. Uno de los más importantes y costosos es el Bakú-Tbilisi-Ceyhan (втс), el cual cuenta con una longitud de $1768 \mathrm{~km}$, y no pasa por territorio ruso o iraní (véase mapa 1). ${ }^{26}$

Asimismo, hay una variedad de gasoductos disponibles en la actualidad para transportar el gas natural, de los cuales destacan: el Blue-Stream, el Gazprom System, el Trans-Balkan, el Korpedzhe a Kurt-Kui (ккк) у el Irán-Turquía. Entre los gasoductos proyectados se encuentran el Bakú-Erzurum, el que financia mayormente BP, el Trans-Afghan Gas Pipeline, que corre desde Turkmenistán hasta Pakistán, y el Trans-Caspian Gas Pipeline (TCGP), que, destinado a satisfacer el mercado europeo, recorrería desde Turkmenistán hasta Turquía (véase mapa 2). ${ }^{27}$

25 MAEC, ficha país citada.

${ }^{26}$ EIA, Caspian countries...

${ }^{27}$ Idem. 


\section{MAPA 1}

\section{Oleoductos de Asia Central}

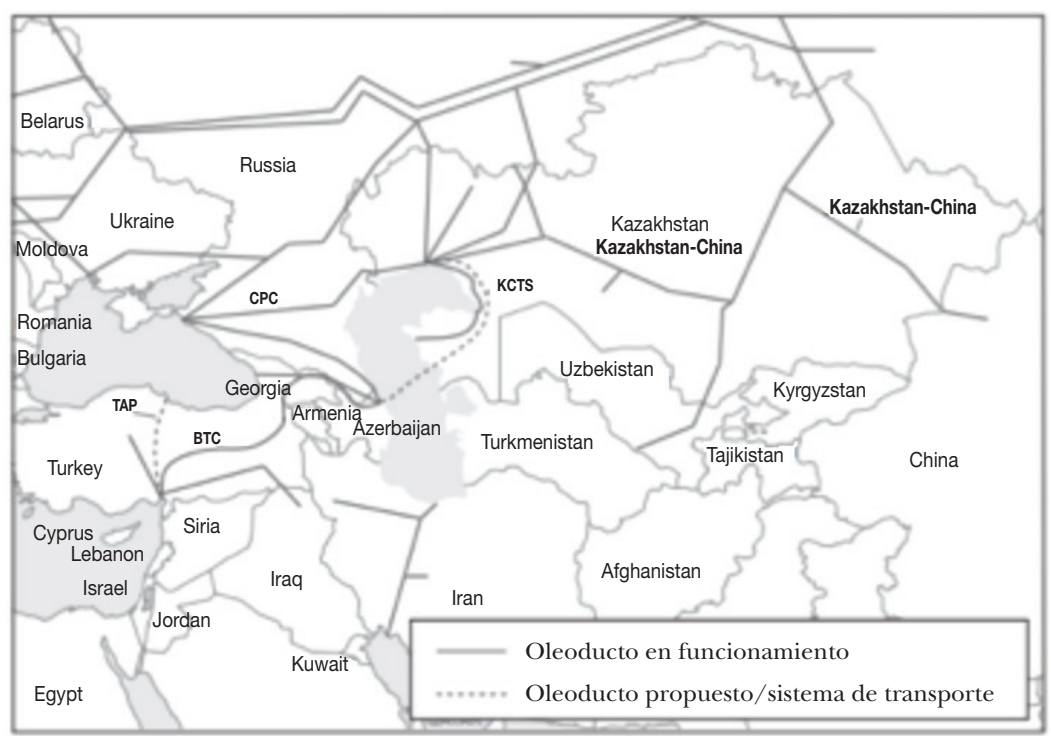

Fuente: R. Jones, “The politics of Central Asia and Caspian Energy", Presentation at Chatham House, Londres, febrero de 2010, en http://www.iea.org/ speech/2010/jones/chatham_house.pdf, consultado en julio de 2016.

Como se puede apreciar, hay una diversidad de tuberías que transportan los hidrocarburos hacia los mercados internacionales. Sin embargo, cada uno de los países de la región está interesado en instalar su propia tubería o al menos querrá que una de éstas pase por su territorio, ya que el estado por donde pase el ducto principal de salida de las producciones del Caspio gozará no sólo de ingresos económicos importantes por la inversión extranjera directa que se implante en la zona, sino que a futuro asegurará para sí una considerable influencia geoestratégica sobre la región.

En un futuro, y dada la importancia de las reservas de gas sobre el total de las reservas de petróleo, todo parece indicar, en fin, que el gas podría tener un papel cada vez más importante en la producción total del Caspio. 


\section{MAPA 2}

\section{Gasoductos de Asia Central}

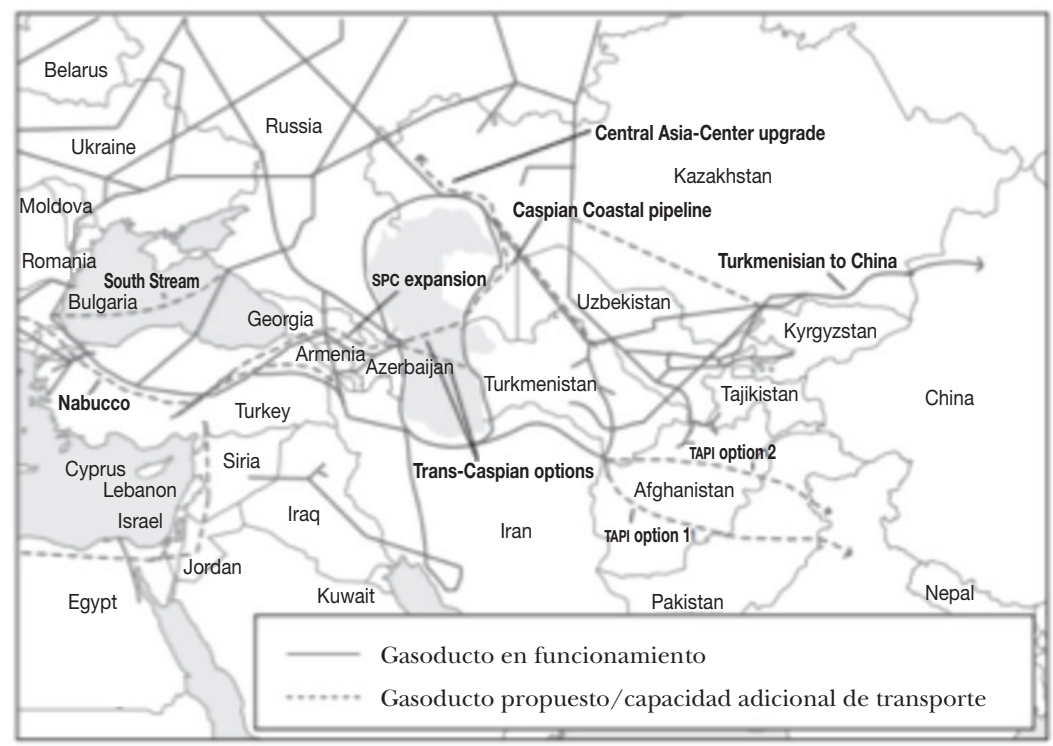

Fuente: Jones, conferencia citada.

\section{Valle de Fergana y sus diferentes efectos en Asia Central}

Hoy en día, el valle de Fergana cubre parte de Kirguizistán, Uzbekistán y Tayikistán. En la época soviética, el valle se dividió entre estas tres repúblicas. Los problemas políticos, demográficos y étnicos derivados de esas divisiones artificiales se hicieron evidentes tras el final de la Unión Soviética, convirtiendo la zona en la de mayor inestabilidad de Asia Central. La zona alberga a unos catorce millones de habitantes, con unos índices de natalidad muy altos; más de la mitad son menores de 18 años; $70 \%$ uzbecos, $20 \%$ kirguices y el resto tayikos. ${ }^{28}$

La competencia por la extracción de petróleo, la industria y la producción agrícola en la zona, han supuesto un choque de

\footnotetext{
28 IEeE, Panorama geopolítico de los Conflictos 2013, Ministerio de Defensa, 2013.
} 
intereses entre Uzbekistán, Kirguistán y Tayikistán. La región cuenta con el 25\% de la producción de algodón uzbeca, pese a suponer sólo el $4,3 \%$ de su superficie total y el $27 \%$ de la población del país, incluidas cinco de las diez ciudades más importantes. Incluye, además, el $75 \%$ de la superficie cultivable tayika y alrededor del $65 \%$ de la producción industrial de este país. ${ }^{29}$

El valle no solamente es objeto potencial de controversias entre las tres repúblicas; es también un lugar de enfrentamientos constantes entre las distintas etnias que lo habitan. Con fronteras geográficamente accidentadas, éstas dividen el valle sin considerar las diferencias étnicas de la región, lo que muchas veces ocasiona conflictos intrarregionales. Por ejemplo, la mayoría de la población que vive en el valle es de origen uzbeko, sin estar en territorio de Uzbekistán. ${ }^{30}$ La presencia de la población uzbeka inquieta a las otras repúblicas (Kirguizistán y Tayikistán), ya que éstas temen que haya movimientos separatistas que decidan anexarse a Uzbekistán. En este caso, el gobierno de Kirguizistán, a cuya población uzbeka tiene por separatista, optó por aislar totalmente esa etnia de la actividad política, lo que deterioró las relaciones con el país vecino, Uzbekistán. ${ }^{31}$

El conflicto suscitado en 2010 en Kirguistán, que puso al país al borde de la guerra civil, supuso un nuevo foco de tensión en la región tras los desplazados que huían del conflicto a la vecina Tayikistán y Uzbekistán por medio del Valle de Fergana. Del mismo modo, se calcula que el $60 \%$ de la población vive bajo el umbral de la pobreza en el valle, mientras que la tasa de paro aproximadamente es del 50\%, lo que obliga a una parte de los habitantes a buscar oportunidades de empleo en el extranjero, especialmente en Rusia. ${ }^{32}$ Además, las relaciones entre los diferentes grupos étnicos se tornan conflictivas debido a las disputas por las tierras, el

${ }^{29} I d$.

${ }^{30}$ Véase "Asia Central”, monográfico de la Revista CIDOB d'Afers Internacionals, núms. 70-71, 2005.

31 A. Khamidov, "Frustration builds among Uzbeks in southern Kyrgyzstan", Eurasianet, 2001, en http://www.eurasianet.org/

32 A. Mahkamov, "Tapping a New Way to Survive", Transitions Online: Regional Intelligence, 2006, en http://www.tol.cz 
agua, la degradación del ambiente y el movimiento de desplazados por la guerra civil en Tayikistán. ${ }^{33}$

Asimismo, a pesar de tener más de dos décadas de vida democrática, hay grandes problemas de corrupción (de 178 países, Tayikistán ocupa, en los índices de corrupción de 2011, el lugar 154; Kazajstán, el 105; Kirguistán, el 164, y Uzbekistán, el 172), nepotismo, ineficiencia, falta de libertades políticas, narcotráfico, terrorismo, degradación ambiental, lo que perturba un proceso de state building inacabado. ${ }^{34}$

El valle de Fergana es el centro islámico de Asia Central, el cual ha resurgido en los últimos años tras la caída del régimen soviético. ${ }^{35}$ La evidencia sugiere que el surgimiento de grupos radicales en la zona se debe a los distintos factores complejos que determinan la vida económica, política y social del Fergana, luego de que los países que integran el valle alcanzaron su independencia. ${ }^{36} \mathrm{La}$ mayoría de estas organizaciones hicieron del Islam su ideología y abogaron por el establecimiento de un estado islámico por medio de diferentes formas, "desde el empleo de métodos no violentos hasta la opción por la yihad armada o la vía militar". ${ }^{37}$

Entre los movimientos islámicos más longevos que surgieron durante este proceso, se encuentra el Movimiento Islámico de

${ }^{33}$ Cf. UNEP, "Environment and Security, Transforming risk into Cooperation: Central Asia/Ferghana/Osh/Khujand area”, UnEP/UndP/Osce/NATO, 2005; IRIN, "Kyrgyzstan: Poverty and migration in the Fergana Valley", 2002, en http:// www.irinnews.org/, consultado en octubre de 2014; y D. Nalle, "Ferghana Report", Central Asia Monitor, núm. 1, 2000, en http:/ /www.friends-partners.org/lists/fergha na-valley/1999/0126.html

${ }^{34}$ IEEE, op. cit.

35 Cf. B. Tursunov y M. Pikulina, "Severe Lessons of Batken", Conflict Studies Research Centre, UK Ministry of Defence, 1999, pp. 1-13; Dyner et al., "Ready to go? ISIS and its presumed expansion into Central Asia”, Policy Paper, núm. 19 (121), junio de 2015, pp. 1-16; e ICG "Syria calling: Radicalisation in Central Asia", Policy Briefing, enero de 2015, pp. 1-15.

36 "Asia Central”, monográfico citado; L.-T. Zapater Espí, "El fundamentalismo islámico en Asia Central”, Cuadernos Const, de la Cátedra Fadrique Furió Ceriol, núms. 41/42, 2002-2003, pp. 181-197; y M. L. Alonso, art. cit.

37 V. Wilches, “Asia Central: Movimientos Islamistas”, 2009, en http://www. rebelion.org/docs/93602.pdf, consultado en noviembre de 2015. 
Uzbekistán (MIU) ${ }^{38}$ y su ramificación, la Unión de la Yihad Islámica y el Hizb ut-Tahrir. Estos grupos fundamentalistas, con liderazgo principalmente uzbeko, se caracterizan por la falta de un proyecto de gobierno, educativo o económico, en beneficio de sus sociedades, tal como lo propone actualmente el Estado Islámico en Irak y Siria (IsIs, por sus siglas en inglés). Sin embargo, son un peligro latente de desestabilización en el valle de Fergana y en la región de Asia Central, ya que durante las últimas dos décadas se han planteado diferentes teorías sobre el probable surgimiento de un escenario más del estilo tribal en la zona y de difícil control.

De Pedro, Dyner et al. e ICG han identificado que desde el año 2011 se ha generado un aumento significativo de los incidentes de violencia relacionados con las actividades de células fundamentalistas en Asia Central. ${ }^{39}$ Asimismo, se ha evidenciado ${ }^{40}$ que el estallido del conflicto sirio y afgano, el surgimiento de isis y la corrupción general, pobreza y mala gestión política en Asia Central y, en especial, en el valle de Fergana, han permitido el reclutamiento de combatientes por parte de IsIs. ${ }^{41}$ Según el ICG las cifras oscilan entre los 2000 y 4000 yihadistas provenientes de las cinco repúblicas de Asia Central, así como de la federación rusa, quienes ven en la religión un vacío generado por la falta de confianza en la política e inseguridad y se enlistan al frente del Estado Islámico. Dyner et al., en su trabajo citado, sugieren que isis no cuenta con la estructura organizacional en Asia Central, pero podría adquirirla, si acepta a MIU en su esfera de influencia, para controlar algunas poblaciones en el valle de Fergana y de ahí esparcirse por toda Asia Central.

${ }^{38}$ Este movimiento ha sido vinculado con el tráfico de armas y drogas en la región y, después del 11/S, con la organización terrorista Al-Qaida, por lo que los gobiernos han utilizado esa vinculación para justificar acciones violentas contra la población musulmana.

${ }^{39}$ N. de Pedro, "Terrorismo yihadista en Asia Central: ¿amenaza existencial o fantasma?”, Opinión CIDOB, núm. 362, octubre de 2015, pp. 1-3; A. Dyner et al., art. cit.; e ICG, art. cit.

${ }^{40} I d$.

${ }^{41}$ Los perfiles de los reclutados son variados, desde jóvenes de 17 años hasta empresarios y mujeres solteras, quienes tienen en mente que el califato islámico podría ser una alternativa seria a la vida postsoviética en la región (id.). 
Definitivamente, el valle de Fergana constituye un punto de tensión extremadamente importante para Asia Central. El futuro de la región dependerá, en gran medida, de la cooperación de las diferentes repúblicas, así como de los nuevos y viejos actores existentes, para solucionar las diferencias por medio del diálogo regional que permita coadyuvar a una mayor integración de la región, para hacer frente a los embates permanentes. En este sentido, se han consolidado y aparecido distintos actores que pretenden ejercer su influencia en la región. A continuación se mencionan los más importantes y sus principales intereses en la zona.

\section{Nuevos y viejos Actores}

La considerable abundancia de recursos naturales y la ubicación geoestratégica de Asia Central han despertado el interés de los principales actores regionales e internacionales, como Rusia, los EE.UU., China, Irán y Turquía. La interferencia de estos actores ha ejercido mayor presión sobre la delicada situación interna de cada país, intensificado las diferencias y rivalidades entre los mismos países de Asia Central y entre algunos vecinos inmediatos y otros lejanos.

\subsection{Federación rusa}

Con la disolución del régimen soviético, Rusia intentó mantener relaciones con las nuevas repúblicas por medio de la Comunidad de Estados Independientes (CEI), ${ }^{42}$ en la cual se planteaba una dinámica de integración de los nuevos países, mientras que otros

${ }^{42}$ La CEI fue fundada el 8 de diciembre de 1991, en Bielorrusia, por un acuerdo entre Ucrania, Bielorrusia y la federación rusa, el cual marca el final de la uRss. El 21 de diciembre del mismo año finalmente once antiguas repúblicas soviéticas (la federación rusa, Armenia, Azerbaiyán, Bielorrusia, Kazajstán, Kirguizistán, Moldava, Tayikistán, Turkmenistán, Ucrania y Uzbekistán, es decir todas excepto Georgia y las tres repúblicas bálticas) adoptaron la Declaración y el Protocolo de Acuerdo de Establecimiento de la Comunidad de Estados Independientes ("Asia Central”, monográfico citado). 
estados veían la CEI como una posibilidad de deslindarse de la federación rusa, que pretendía seguir siendo el actor dominante en Asia Central. ${ }^{43}$ Asimismo, dada la posición estratégica que goza la región, Rusia comprendió que tenía que proteger su seguridad nacional y evitar cualquier influencia y penetración estratégica de otra potencia regional, como podrían ser Irán, China, Pakistán, Turquía o, probablemente, los EE.UU.

Todo parece indicar que Rusia sigue considerando a Asia Central como parte importante de su perímetro de seguridad, por lo que Moscú ha puesto su atención en la continuidad de sus lazos militares con las diferentes repúblicas exsoviéticas. ${ }^{44}$ Sin embargo, esta estrategia no ha sido tan exitosa como se esperaba. Si bien tiene garantizada la presencia militar en Tayikistán, Kazajstán y Kirguistán, la situación en Uzbekistán es diferente, ya que el gobierno de Tashkent también tiene ambiciones territoriales en la región y ha rechazado el establecimiento de bases rusas en su país. ${ }^{45}$ A pesar de que Uzbekistán ingresó a la Organización del Tratado de la Seguridad Colectiva (OTSC), lo que la convertía en aliada militar de Moscú, determinó abandonar el bloque en 2008 para fijar la mirada en Occidente.

En el aspecto económico, sin lugar a dudas, Asia Central depende más de Rusia que a la inversa. Sin embargo, después de la independencia de las repúblicas, la influencia económica de Rusia ha disminuido en la región por la entrada de nuevos competidores, en especial de China, y por la falta de una visión capitalista. En este sentido, la política rusa se ha orientado hacia el aspecto militar y lucha contra el terrorismo más que por el progreso de los vínculos económicos, a pesar de que gozaba de ventajas de las cuales pudo sacar provecho. ${ }^{46}$

${ }^{43} \mathrm{Id}$.

${ }^{44}$ Cf. Ch. Fairbanks et al., Strategic Assessment of Central Eurasia, Washington, DC, The Atlantic Council of the United States, 2001, y G. W. Lapidus, "Central Asia in Russian and American Foreign Policy after September 11, 2001”, Presentation from the panel discussion on Central Asia and Russia: "Responses to the War on Terrorism”, Berkeley, University of California, 2001.

${ }^{45}$ S. Akbarzadeh, "Keeping Central Asia stable", Third World Quarterly, vol. 5, núm. 4, 2004, pp. 689-705, y Roy, op. cit.

${ }^{46}$ A. Vassiliev, Central Asia, Political and Economic Challenges in the Post-Soviet Era, Londres, Saqi Books, 2001. 
En la actualidad, Moscú ha puesto en marcha, desde 2010, un bloque económico entre los países del antiguo régimen soviético. Éste, que entró en vigor el primero de enero de 2015, está conformado por Rusia, Bielorrusia, Kazajstán, Armenia y Kirguistán. Este proyecto de integración en el espacio postsoviético pretende agrupar a las antiguas repúblicas soviéticas en la Unión Económica Eurasiática (UEE), la cual tiene su base en la Unión Aduanera. La UEE procura ser un organismo más sólido emanado de la CEI, en el cual se pretende impulsar el tránsito de mercancías, capitales y mano de obra entre sus miembros, así como políticas comunes en materia económica.

En ese sentido, se han establecido las bases para que la Unión contemple acuerdos preferenciales con países como China, India, Vietnam e Israel, y con países latinoamericanos como Venezuela, Argentina, Chile y Perú. Este proceso de integración contempla nuevas posibilidades de desarrollo para los Estados de Asia Central, ya que, según los principios de integración económica, se garantiza el reparto equitativo de los recursos, autonomía y coordinación entre sus miembros. El propósito radica en frenar la escalada de tensión que se vive en el espacio postsoviético desde la desintegración de la URSs, además de frenar la influencia de otros países en la región.

\subsection{Los Estados Unidos de América}

Cuando los Ee.uU. enviaron sus tropas a Asia Central en el año 2001, se terminó con una política pasiva respecto a las repúblicas exsoviéticas. Durante la década de 1990, Washington aceptó la posición rusa de que Asia Central era parte de su círculo de influencia o el backyard ruso. En términos estratégicos, los EE.UU. consideraban que la región estaba fuera del alcance de su dominio, además de tomar en cuenta la posibilidad de que se dañarían las relaciones con Rusia, si se pretendía algún tipo de intervención. La región centroasiática ofrecía abundantes recursos naturales, por lo que Washington sumó esfuerzos para la integración de las nuevas economías al mercado mundial, especialmente por medio de industrias extractivas de petróleo y gas, con la finalidad de reducir la 
dependencia de hidrocarburos procedentes del Golfo Pérsico, así como también con la intención de "promover la estabilidad, soberanía y democracia en la nuevas repúblicas". ${ }^{47}$ Sin embargo, en el aspecto económico, este objetivo se llevaría a cabo con la participación de empresas privadas y organizaciones internacionales, en las que el gobierno norteamericano tendría capacidades limitadas de intervención. ${ }^{48}$ No obstante, los EE.UU. han procurado intervenir en la región, para que la construcción de los nuevos oleoductos no pase por territorio anti-norteamericano (Rusia, Irán y China), y, de esa manera, garantizar a las empresas y consorcios con capital estadounidense la salida de los hidrocarburos a los mercados, principalmente de Afganistán, Sureste y Este asiático y Europa. ${ }^{49}$

Después de los ataques del 11/S, la suposición de que Asia Central se encontraba geográficamente muy lejana se desvanecía, ya que la inestabilidad de la región y el apoyo a grupos terroristas por parte de algunos gobiernos locales amenazaban directamente la seguridad nacional de los EE.uU. Por ello, se puso en marcha la guerra internacional contra el terrorismo, primero en Afganistán y después en Irak, lo que supuso que la geografía no sería más un factor estratégico determinante, mientras se tratase de salvaguardar la seguridad del país. En este sentido, Stephen J. Blank argumenta que la nueva política de Washington en Asia Central es la de "crear nuevas relaciones con Estados que son fundamentales para la guerra en contra del terrorismo" ${ }^{50}$ y tener acceso militar norteamericano permanente en la zona. ${ }^{51}$

${ }^{47}$ E. Rumer et al., U.S. Policy toward Central Asia 3.0, Washington, DC, Carnegie Endowment for International Peace, Publication Department, 2016, p. 1.

48 Akbarzadeh, art. cit.

${ }^{49}$ Rumer et al., op. cit., p. 19. Cabe señalar que las importaciones de hidrocarburos de los EE.Uu., procedentes de la región de Asia Central en 2014, fueron principalmente de Kazajstán con un valor de 347 millones de dólares, una cifra poco significativa en términos geoestratégicos. Véase uscB, "U. S. International Trade in Goods and Services (FT900), Exhibit 17a- U.S. Imports of Crude Oil by Selected Countries", 2016, en https://www.census.gov/foreign-trade/Press-Release/current_press_release/index.html, consultado en julio de 2016.

50 "After Two Wars: Reflections on the American Strategic Revolution in Central Asia”, Conflict Studies Research Centre, Central Asia Series, 05/14, 2005, p. 1.

${ }^{51}$ A cambio del acceso militar, Washington puso a disposición ayuda financie- 
A pesar de los esfuerzos de los EE.UU. de poner freno a la amenaza del terrorismo en la región de Asia Central, al firmar acuerdos con algunas de las extintas repúblicas soviéticas, éstos han sido socavados por la presión del Kremlin. Hoy en día el interés integracionista propuesto por Rusia y respaldado por otras cinco repúblicas soviéticas extintas (Armenia, Bielorrusia, Kazajstán, Kirguistán y Tayikistán) resulta en la disminución de la influencia de Washington en la región de Asia Central y, por tanto, en la expulsión total de sus bases militares de la zona. ${ }^{52}$ Sin embargo, es preciso señalar que los cambios geopolíticos en Asia Central han ido mermando el poder de los EE.UU. en la región, y actualmente hay otras potencias regionales (China, Irán y Turquía) que intentan influir en la zona según sus intereses particulares. Asimismo, la dinámica interna en Asia Central está en una trayectoria diferente, en la que hay un cambio geoestratégico para disminuir sus lazos con la comunidad euroatlántica y aumentar su influencia con China. Lo anterior impone un nuevo reto a los EE.UU. con la finalidad de mantener sus alianzas con los países centroasiáticos.

\subsection{China}

En los últimos años, China se ha convertido en un actor importante en el mundo y, en especial, en la escena de Asia Central. Los

ra y logística a cada una de las repúblicas centroasiáticas. Asimismo, se firmaron varios acuerdos de cooperación tecnológica militar con Tayikistán, Kirguizistán y Uzbekistán, que prevén ejercicios militares conjuntos, donación de equipos y apoyo logístico, así como entrenamientos e intercambio de informaciones militares (J. Nichol, "Central Asia's Security: Issues and Implications for U.S. Interests", CRS Report for Congress, Washington, DC, U.S. Department of State, 2006).

${ }^{52}$ Los EE.UU. mantuvieron dos bases militares en la región con el objetivo de apoyar las acciones de la posguerra en Afganistán; una ubicada al sur de Uzbekistán, la base aérea de Karshi-Khanabad (2001-2005), y la otra, al norte de Biskek, la capital de Kirguistán, la base aérea de Manas (2001-2014). Cf. L. Beehner, ASIA: U.S. Military Bases in Central Asia, Council on Foreign Relations background Q \& A, 26 de julio, 2005, en http://www.cfr.org/russia-and-central-asia/asia-us-military-bases-central-asia/p8440\# consultado en julio de 2016, y U.S. Air Force, "Locations", 2016, en https:/ / www.airforce.com/lifestyle/locations, consultado en julio de 2016. 
intereses de Pekín en la zona giran principalmente alrededor de tres ejes: el intercambio comercial, el abastecimiento de hidrocarburos y la seguridad nacional. Para materializar estos objetivos, el gobierno chino, junto con los ruso, kazajo, kirguizo y tayiko, crearon en 1996 la Shangai Cooperation Organization (sco). Uzbekistán se integraría más tarde en el año 2001, mientras que Pakistán, India e Irán se incorporarían como observadores en julio de $2005 .^{53}$

En el aspecto comercial, desde la desintegración de la Unión Soviética, China ha ido incrementando sus relaciones económicas con los vecinos centroasiáticos. ${ }^{54} \mathrm{~A}$ la inversa de Rusia, que insistió en influir militarmente en la región, Pekín ha utilizado la expansión de las actividades económicas y el aumento de sus inversiones en Asia Central, para reforzar la estabilidad de la zona y su influencia.

En sus relaciones comerciales con la región, China cuenta con un cierto número de ventajas sobre los demás competidores, ya que la proximidad cultural de su provincia de Xinjiang con la región facilita el comercio chino-centroasiático, pero también contribuye a complementar ambos mercados. El mercado chino presenta buenas oportunidades para los productos y materias primas de Asia Central, mientras que las repúblicas de la región se interesan por los bienes de consumo, productos agrícolas e industriales y en los últimos años el gobierno chino ha introducido su maquinaria, productos electrónicos y desarrollado avanzada tecnología. ${ }^{55}$

En lo que atañe al abastecimiento de hidrocarburos, EIA estima que el crecimiento del consumo de petróleo de China ha disminuido por la actual crisis financiera mundial, después de haber crecido de manera anual el $11 \%$ en 2010 . Sin embargo, a pesar de la desaceleración en el consumo del petróleo, el país aún representaba

53 Véanse E. Barrera, La política exterior de los Estados Unidos en Asia Central: el caso de Uzbekistán, México, Escuela de Relaciones Internacionales, Universidad Anáhuac, 2006; S. Atal, "The New Great Game”, The National Interest, otoño 2005, pp. 101-105; y MFA, "Shanghai Cooperation Organization", 2004, en http://www. fmprc.gov.cn/eng/topics/sco/t57970.htm

${ }^{54}$ Cf. S. Atal, art. cit.

${ }^{55}$ N. Swanström, "Chinese Business Interests in Central Asia: A Quest for Dominance”, Central Asia-Caucasus Analyst, 18 de junio, 2003. 
más de un tercio del crecimiento de la demanda mundial de éste en 2014. ${ }^{56}$ La mayor parte de esos hidrocarburos procede del Golfo Pérsico, lo que plantea un problema importante de seguridad para China, dadas sus crecientes necesidades de petróleo. Esto ha motivado una estrategia de diversificación para el acceso a nuevos recursos petrolíferos, como serían los países de Asia Central. ${ }^{57}$

Es vital una diversificación de sus fuentes de suministros petrolíferos para reducir su dependencia del Pérsico y la vulnerabilidad estratégica que resulta de ello, al no disponer de la capacidad necesaria para garantizar la protección de las vías marítimas entre el Golfo y el mar de China Meridional en caso de dificultades con los EE.uU. Por tanto, China tiene interés en que las riquezas de Asia Central no sean explotadas solamente para beneficio de los países europeos y en que Rusia no controle las vías de salida de los hidrocarburos, por lo que en 2006 se puso en marcha un oleoducto que bombea directamente desde Kazajstán hasta la región de Xinjiang (Kazakhstan-China Oil Pipeline), así como la puesta en marcha en 2009 de un gasoducto que suministra gas natural de Turkmenistán a China a través de la región de Xinjiang (Central Asia-China Gas Pipeline) (véanse Mapas 1 y 2). 58

\subsection{República Islámica de Irán}

Por su posición geográfica, Irán constituye una de las rutas más cortas, simples, rápidas y baratas para sacar los hidrocarburos del Caspio al mercado internacional. El resto de las rutas son más complicadas económica y geográficamente. ${ }^{59}$ Dar salida a la producción del Caspio a través del Golfo Pérsico daría a los países centroasiáticos la oportunidad de acercarse a sus futuros mercados potenciales en India, Pakistán, China o Japón. Sin embargo,

56 "China, international energy and data analysis", 2015a, en https://www.eia. gov/beta/international/analysis.cfm?iso=CHN, consultado en noviembre de 2015 .

${ }^{57} \mathrm{Id}$.

${ }^{58} \mathrm{Id}$.

${ }^{59}$ S. T. Hunter, Central Asia since independence, Londres, Preager Publishers, 1996. 
significaría privar también a los países consumidores de Europa Occidental de la posibilidad de diversificar su mercado de aprovisionamiento y expondría los hidrocarburos del Caspio a los mismos riesgos de producción que los del Golfo Pérsico.

Entre los obstáculos que Irán debe superar para ser un protagonista en Asia Central figuran: su limitado poder económico y el atraso tecnológico que no permite influir ni convencer económicamente a los países centroasiáticos; su ideología antiamericana y antiisraelí, que lo mantendrá al margen como foco de influencia en la región. A pesar de haberse alcanzado un acuerdo nuclear en $2015^{60}$ entre Teherán y Occidente, para desistir de estos planes, las secuelas de las sanciones impuestas aún están latentes en el país persa. Por último, el aislamiento al que se enfrentó Irán por su empeño en seguir adelante con su programa nuclear, que impide la edificación de una política centroasiática por parte de su gobierno.

La entrada de Irán en la sco como observador en el año 2005, permite a China reforzar su posición en contra de los EE.UU. en la organización, por lo que se vislumbra la formación de un bloque centroasiático antinorteamericano. Sin embargo, en la misma organización hay aliados de los EE.uU. que pueden equilibrar la balanza. También, permite crear una política común referente a la energía de Asia Central y sus rutas de salida, al menos con los demás miembros del grupo, excepto Rusia.

\subsection{Turquía}

Después de la caída del bloque soviético, se esperaba que Turquía tuviera una participación más activa en el territorio centroasiático por el gran número de población turcófona que vive en la región. Sin embargo, la creación de un espacio "panturco" ha fracasado, principalmente por la diferencia de intereses entre los distintos

${ }^{60}$ Para profundizar en el acuerdo alcanzado entre Irán y el sexteto (EE.UU., Rusia, Reino Unido, China, Francia y Alemania) para reducir su programa nuclear, se puede consultar el siguiente enlace: https://actualidad.rt.com/actualidad/180 071-detalles-acuerdo-nuclear-iran-sexteto 
países y por la negativa de los gobiernos centroasiáticos de estar bajo la influencia de un país líder. ${ }^{61}$ Con todo, los intereses turcos van más allá de la creación de un espacio turcófono en Asia Central. Su principal interés es económico y está relacionado con los hidrocarburos.

Turquía ha dado mayor importancia al desarrollo de la energía. Se ha concentrado en la creación de una vía este-oeste (apoyada principalmente por Inglaterra y los EE.UU.) la distribución de los hidrocarburos del mar Caspio. Esta vía, conocida como la tubería Bakú-Tbilisi-Ceyhan (BTC Pipeline), procede desde el puerto de Bakú en Azerbaiyán, pasando por Georgia y el este de Turquía, hasta llegar a la terminal mediterránea de Ceyhan. El gobierno de Ankara ha puesto especial interés en el BTC, para ser un país de tránsito más que un país comprador. Además, el oleoducto puede llegar a ser un punto a favor de Turquía, para otorgarle una posición geoestratégica a Ankara en su posible entrada como miembro a la UE, donde se podría asegurar el acceso europeo a los recursos asiáticos que cruzaran por el territorio turco. ${ }^{62}$

En lo que concierne al gas, Turquía cuenta con diferentes terminales de gas licuado y múltiples conexiones con ductos que le permiten tener una relativa diversidad de suministro, pese a lo cual, depende aún del gas ruso en un 57\%. ${ }^{63}$ Asimismo, las importaciones de gas natural con frecuencia se ven afectadas o suspendidas por ataques terroristas contra los gasoductos o incluso por inclemencias climáticas, lo cual ha forzado al gobierno turco a buscar mayores y más seguros suministros de gas, concentrándose en los diferentes mercados centroasiáticos, principalmente en el de Turkmenistán. ${ }^{64}$

${ }^{61}$ Roy, op. cit.

${ }^{62}$ Hasta junio de 2016 esta estrategia turca no ha sido factor para su inclusión como miembro de la Unión Europea. Sin embargo, Turquía continúa cooperando con Europa en otros ámbitos, como la crisis de refugiados sirios y otros problemas que afectan la seguridad de la uE, con la finalidad de ingresar, en un futuro, al bloque de integración económica más avanzado del mundo.

${ }^{63}$ EIA, "Turkey, international energy and data analysis", 2015b, en https:/ / www. eia.gov/beta/international/analysis.cfm?iso=TUR, consultado en julio de 2016 .

${ }^{64} \mathrm{Id}$. 
La posición estratégica que tiene Turquía podría ser aún mayor, si finalmente tuviera acceso a la uE. Podría añadir una nueva dimensión a los esfuerzos en política exterior de la UE en regiones de vital importancia como Oriente Medio, el Mediterráneo, Asia Central y el sur del Cáucaso. En este plano, la uE ha tenido un papel menor en las repúblicas centroasiáticas, pero Turquía, por razones de geografía, cultura, religión e idioma, podría tener un papel más activo, para expandir la democratización de los países islámicos en la región. Asimismo, su participación militar en Afganistán, bajo la insignia de la Organización del Tratado del Atlántico Norte (otan), confirma que es un fiel aliado de Occidente y que puede influir en la zona a favor de la OTAN y de la UE. ${ }^{65}$

\section{Conclusiones}

La innegable relevancia que la conformación del bloque centroasiático aporta a la esfera mundial estriba básicamente en dos factores: su privilegiada ubicación geográfica y los factores políticos que de su incipiente creación emanan. En este sentido, no es de extrañar que el surgimiento de los denominados Cinco-stan sea objeto de los intereses políticos de las potencias mundiales plenamente consolidadas.

La desaparición de un mando único de gobierno y la aparición de nuevos estados con intereses particulares explican la diversidad descrita de conflictos que amenazan la estabilidad presente y futura de Asia Central. Sin embargo, por la complejidad del reordenamiento geopolítico regional y la velocidad con que se transforman los acontecimientos en esta época globalizada resulta arriesgado vaticinar el futuro de la región. Sin embargo, destacan dos acontecimientos fundamentales que pueden ayudar a comprender los conflictos centroasiáticos, así como vislumbrar una posible solución futura.

La incorporación en el escenario internacional de los países centroasiáticos tras la caída del modelo soviético se ha mostrado

${ }^{65}$ Blank, art. cit. 
(o por lo menos lo ha intentado) bajo un esquema de gobierno con tendencia más democrática que sus antecesores, tentativa que a su vez ha transformado la geopolítica de la región mediante decisiones y consecuencias que trascienden las fronteras mismas del heartland. Desde su independencia, las nuevas repúblicas han experimentado en el ámbito interno una diversidad de conflictos y retos relacionados con la construcción y consolidación de un estado-nación, en que se han encaminado a la búsqueda de un nuevo sistema alternativo que remplace la filosofía comunista de la sociedad, de las instituciones y de los gobernantes. En general, su incursión en la búsqueda de un modelo propio de gobierno ha sido poco exitosa, pues han pasado de un totalitarismo a un autoritarismo heredado dentro de sus formas de gobierno donde aún es evidente la necesidad fundamental de consolidar los derechos y el desarrollo humano junto con el crecimiento económico, como elementos base deseables para inducir el progreso democrático de estas naciones. En el nuevo papel protagónico sociopolítico interno y hasta en tanto se consolide una visión propia de nación es explicable la proliferación de diferentes grupos afines que procuran su hegemonía en los estados y en la región, con los riesgos que conllevan los enfoques radicales de algunos de ellos, que en casos extremos pueden ser objeto de la condena mundial.

El caso particular del conflicto sobre los derechos y usos de los recursos del Mar Caspio reflejan, en gran medida, la inestabilidad de la región por los diferentes intereses que persiguen los países ribereños respecto a las reservas de gas y petróleo en el subsuelo marino. Los nuevos estados perciben el acceso a estos recursos como la única base para su desarrollo económico a corto y mediano plazos de la mano de inversiones occidentales, lo cual propicia una gran tensión política con Rusia e Irán, que tenían el uso exclusivo del mar antes de la independencia de Asia Central y el Cáucaso. Aunado a lo anterior, dada la importancia de las reservas de gas y en menor medida de petróleo, que se han estimado en los últimos años para la zona de Asia Central, se prevé que habría cada vez más una mayor competencia entre los países de la región para fijar sus alianzas con Oriente u Occidente, para la planeación del 
trayecto o salida de los distintos ductos y así asegurar una posición geoestratégica en la región.

El conflicto étnico y el incremento del fundamentalismo islámico, especialmente en el Valle de Fergana, son punto de tensión política, económica y social, cuya alteración afectaría a Kirguistán, Tayikistán y Uzbekistán, pudiendo ser utilizados por las fuerzas extremistas, especialmente Isis, para ampliar su presencia en la región. Lo anterior implicaría una mayor intervención de Rusia por medio de la отSC y de manera conjunta Rusia y China con la SCO, pero también de los EE.UU. para impedir una mayor inestabilidad en Afganistán, además de inhibir cualquier amenaza para la seguridad interna de los EE.UU.

En este sentido, la estabilidad de toda la zona dependerá en gran medida de la solución política y pacífica de las controversias entre las repúblicas centroasiáticas y una mayor cooperación de los nuevos y viejos actores. De trascendencia equivalente al surgimiento de las nuevas repúblicas es la participación directa por primera vez en la historia de los EE.UU. en el corazón de Asia, situación que ha enarbolado el resurgimiento de la región como el "pivote del mundo”. Así como los ataques del 11/S fueron inéditos en el mundo, sus consecuencias no han tenido precedentes. La entrada de los EE.UU. en el backyard ruso ha desequilibrado el status quo entre los diferentes actores que pretendían influir en la región. Su presencia en Asia Central ha sido incómoda para las potencias regionales, en especial para China y Rusia, que temen por el unilateralismo norteamericano. Tan evidente es el anterior razonamiento que como respuesta al intervencionismo estadounidense, China ha intentado crear un polo opositor antinorteamericano mediante la SCT, para fomentar el multilateralismo y quitar protagonismo a los EE.Uu., mientras que Rusia, por su parte, ha ejercido presión en algunos países centroasiáticos (por medio del proyecto de integración económica regional que pretende aglutinar a las repúblicas soviéticas extintas en la Unión Económica Euroasiática, así como política y militarmente por la OTSC), para hacer un contrapeso a la presencia estadounidense en la región con la clara intención de minimizar la presencia de Washington en la zona. Lo cierto es que, más allá de los esfuerzos de Rusia y China por minimizar la 
capacidad de influencia de los EE.UU. en la región, hay evidencia ${ }^{66}$ que sugiere un nuevo replanteamiento de la participación de los EE.UU. en Asia Central menos intrusiva y cada vez más cooperativa con Rusia y China (que han ido tomando el liderazgo económico y militar en la zona) y así aprovechar las acciones de éstos para promover los intereses de los EE.UU.

El resto de potencias regionales, como Turquía e Irán, continuarán consolidando sus fortalezas, tanto para tener un papel protagónico en Asia Central, como para atraer capitales extranjeros para el desarrollo de nuevos ductos que den salida a los hidrocarburos de la región. La posición geográfica de Asia Central ofrece a las potencias mundiales y regionales interesadas en la zona una posición estratégica que les permite un amplio margen de acción y control sobre una de las zonas más influyentes en el mundo (principalmente por su capacidad de producción de hidrocarburos), por lo que la resolución de sus conflictos, ya sea de manera individual o conjunta, serán trascendentales para la geopolítica mundial.

\section{Bibliografía}

Akbarzadeh, S., "Keeping Central Asia stable", Third World Quarterly, vol. 5, núm. 4, 2004, pp. 689-705.

Alonso, María Luciana, "Fundamentalismo Islámico en Asia Central: causas, actores y perspectivas de futuro", Grupo de Estudios Internacionales Contemporáneos, AI 014/2011, Asia-Pacífico, 14 de julio de 2011, pp. 3-14.

"Asia Central", monográfico de la Revista CIDOB d'Afers Internacionals, núms. 70-71, 2005.

Atal, Subodh "The New Great Game", The National Interest, otoño 2005, pp. 101-105.

Barrera, E., La política exterior de los Estados Unidos en Asia Central: el caso de Uzbekistán, México, Escuela de Relaciones Internacionales, Universidad Anáhuac, 2006.

Beehner, Lionel, ASIA: U.S. Military Bases in Central Asia, Council on Foreign Relations background Q \& A, 26 de julio, 2005, en http://www.

${ }^{66}$ Cf. Rumer et al., op. cit. 
cfr.org/russia-and-central-asia/asia-us-military-bases-central-asia/ p8440\#

Blank, Stephen J., "After Two Wars: Reflections on the American Strategic Revolution in Central Asia”, Conflict Studies Research Centre, Central Asia Series, 05/14, 2005.

BP, "Statistical Review of World Energy", junio de 2014, en http://www. bp.com/content/dam/bp/pdf/Energy-economics/statistical-review-2014/BP-statistical-review-of-world-energy-2014-full-report.pdf

"Cuarta Cumbre del Caspio", Kremlin, Astrakhan, 2014, en http://kremlin.ru/news $/ 46686$

Cutler, R. M., "Developments in the evolving Caspian legal regime: part 2-2", FSU Oil E̋ Gas Monitor, núm. 97, 2000, pp. 4-6, en http://www. gasandoil.com/goc/news/ntc03767.htm

De Pedro, Nicolás, "Terrorismo yihadista en Asia Central: ¿amenaza existencial o fantasma?", Opinión CIDOB, núm. 362, octubre de 2015, pp. 1-3.

Dyner, Anna, Arkadiusz Legiec y Kacper Rekawek, "Ready to go? ISIS and its presumed expansion into Central Asia”, Policy Paper, núm. 19 (121), junio de 2015, pp. 1-16.

Djalili, M. R. y T. Keller, "Regímenes políticos de Asia Central: crisis de legitimidad, violencia política y perspectivas inciertas”, Revista CIDOB d’Afers Internacionals, núms. 70-71, 2005, pp. 35-68.

EIA: Energy Information Administration, Caspian Sea, Turkey, India, China, Central Asia, Washington, DC, Country Analysis Briefs, 2005.

- Overview of oil and natural gas in the Caspian Sea region, Caspian Sea series, 26 de agosto, 2013a.

, Caspian countries are developing new oil and natural gas export capacity, 2013b, en http://www.eia.gov/todayinenergy/detail.cfm?id=12931

—, "China, international energy and data analysis", 2015a, en https://www.eia.gov/beta/international/analysis.cfm?iso=CHN

— https://www.eia.gov/beta/international/analysis.cfm?iso=TUR

, Beta: International, Reserves and Capacity, Petroleum and Natural Gas, 2016, en http://www.eia.gov/beta/international/

Fairbanks, Charles, S. Frederick Starr, C. Richard Nelson y Kenneth Weisbrode, Strategic Assessment of Central Eurasia, Washington, DC, The Atlantic Council of the United States, 2001. 
Hunter, Shireen T., Central Asia since independence, Londres, Preager Publishers, 1996.

IEeE: Instituto Español de Estudios Estratégicos, Panorama geopolítico de los Conflictos 2013, Ministerio de Defensa, 2013.

ICG: International Crisis Group, "Syria calling: Radicalisation in Central Asia”, Policy Briefing, enero de 2015, pp. 1-15.

IRIN: Integrated Regional Information Networks, "Kyrgyzstan: Poverty and migration in the Fergana Valley", 2002, en http:/ /www.irinnews.org/

Jones, R., "The politics of Central Asia and Caspian Energy", Presentation at Chatham House, Londres, febrero de 2010, en http://www.iea. org/speech/2010/jones/chatham_house.pdf

Khamidov, Alisher, "Frustration builds among Uzbeks in southern Kyrgyzstan”, Eurasianet, 2001, en http://www.eurasianet.org/

Kovalskii, V. F., "Democratic Declarations and Political Realities", en A. Vassiliev (ed.), Central Asia, Political and Economic Challenges in the PostSoviet Era, Londres, Saqi Books, 2001.

Laherrere, J., "Forecasting future production from past discovery", $O P E C$ Semminar, septiembre 28-29, Viena, 2001.

Lapidus, Gail W., "Central Asia in Russian and American Foreign Policy after September 11, 2001", Presentation from the panel discussion on Central Asia and Russia: "Responses to the War on Terrorism", Berkeley, University of California, 2001.

Mackinder, H. J., "The Geographical Pivot of History", The Geographical Journal, vol. 23, núm. 4, 1904, pp. 421-437.

Mahkamov, Anvar, "Tapping a New Way to Survive", Transitions Online: Regional Intelligence, 2006, en http:/ / www.tol.cz

Mañé, A., "Territorios ricos en hidrocarburos de Asia Central, ¿países productores, enclaves exportadores o países de tránsito?", Revista CIDOB d'Afers Internacionals, núms. 70-71, 2005, pp. 87-113.

maEc: Ministerio de Asuntos Exteriores y de Cooperación, "Turkmenistán, ficha país”, Gobierno de España, Oficina de Información Diplomática, 2013.

-, "Tayikistán, ficha país", Gobierno de España, Oficina de Información Diplomática, 2013.

MFA: Ministry of Foreign Affairs of the People's Republic of China, "Shanghai Cooperation Organization", 2004, en http://www.fmprc. gov.cn/eng/topics/sco/t57970.htm 
Nalle, David, "Ferghana Report", Central Asia Monitor, núm. 1, 2000, en http://www.friends-partners.org/lists/ferghana-valley/1999/0126. html

National Energy Policy Development Group, Reliable, Affordable, and Environmentally Sound Energy for America's Future, Washington, DC, United States Government Printing, 2001.

Nichol, Jim, "Central Asia's Security: Issues and Implications for U.S. Interests", CRS Report for Congress, Washington, DC, U.S. Department of State, 2006.

ONU: Organización de las Naciones Unidas, Convención de las Naciones Unidas sobre el Derecho del Mar, 2006, en www.un.org/depts/los/convention_agreements/texts/unclos/convemar_es.pdf

Pérez Martín, Miguel Ángel, "El status jurídico del Mar Caspio”, Geoeconomics and Geopolitics Journal, 1994.

Petrov, N. I., "Political Stability in the Conditions of the Command-Administrative Regime", en A. Vassiliev (ed.), Central Asia, Political and Economic Challenges in the Post-Soviet Era, Londres, Saqi Books, 2001.

Roberts, J., "Caspian oil and Gas, How far have we come and where are we going?”, en Sally N. Cummings (ed.), Oil, Transition and Security in Central Asia, Londres, Routledge Curzon, 2003.

Roy, O., La nueva Asia Central o la fabricación de naciones, Madrid, Sequitur, 1997.

Rubin, Barnett R., "Central Asia Wars and Ethnic Conflicts, Rebuilding Failed States”, Human Development Report Office, United Nations Development Programme, 2004.

Rumer, Eugene, Richard Sokolsky y Paul Stronski, U.S. Policy toward Central Asia 3.0, Washington, DC, Carnegie Endowment for International Peace, Publication Department, 2016.

Sainz, Nora, "Asia Central en un mundo en cambio: de región periférica a área generadora de aplicaciones de políticas", Revista CIDOB d'Afers Internacionals, núms. 70-71, 2005, pp. 115-141.

Swanström, Niklas, "Chinese Business Interests in Central Asia: A Quest for Dominance", Central Asia-Caucasus Analyst, 18 de junio, 2003.

Tursunov, Bakhrom y Marina Pikulina, "Severe Lessons of Batken”, Conflict Studies Research Centre, UK Ministry of Defence, 1999, pp. 1-13.

UNEP: United Nations Environmental Programme, "Environment and Security, Transforming risk into Cooperation: Central Asia/Ferghana/ 
Osh/Khujand area”, United Nations Environmental Programme/ United Nations Development Programme/Organization for Security and Co-operation in Europe/North Atlantic Treaty Organization, 2005.

UNODC: United Nations Office on Drugs and Crime, “Afganistán: UNODC advierte riesgo de que se convierta en «narco-Estado»", Centro de noticias onU online, 28 de junio de 2006. , "Afghanistan Opium Survey", Viena, 2013.

, "Informe mundial sobre las drogas 2014", Viena, 2014.

U.S. Air Force, "Locations", 2016, en https:/ / www.airforce.com/lifestyle/ locations

USCB: U.S. Census Bureau, "U. S. International Trade in Goods and Services (FT900), Exhibit 17a- U.S. Imports of Crude Oil by Selected Countries", 2016, https://www.census.gov/foreign-trade/Press Release/ current_press_release/index.html

Vassiliev, A., Central Asia, Political and Economic Challenges in the Post-Soviet Era, Londres, Saqi Books, 2001.

Wilches, Víctor, “Asia Central: Movimientos Islamistas”, 2009, en http:/ www.rebelion.org/docs/93602.pdf

Zapater Espí, Luis-Tomás, "El fundamentalismo islámico en Asia Central”, Cuadernos Const, de la Cátedra Fadrique Furió Ceriol, núms. 41/42, 2002-2003, pp. 181-197. 